\title{
Anaerobic microbial LCFA degradation in bioreactors
}

\author{
D. Z. Sousa, M. A. Pereira, J. I. Alves, H. Smidt, A. J. M Stams \\ and M. M. Alves
}

\begin{abstract}
This paper reviews recent results obtained on long-chain fatty acids (LCFA) anaerobic degradation. Two LCFA were used as model substrates: oleate, a mono-unsaturated LCFA, and palmitate, a saturated LCFA, both abundant in LCFA-rich wastewaters. 16S rRNA gene analysis of sludge samples submitted to continuous oleate- and palmitate-feeding followed by batch degradation of the accumulated LCFA demonstrated that bacterial communities were dominated by members of the Clostridiaceae and Syntrophomonadaceae families. Archaeal populations were mainly comprised of hydrogen-consuming microorganisms belonging to the genus Methanobacterium, and acetate-utilizers from the genera Methanosaeta and Methanosarcina. Enrichment cultures growing on oleate and palmitate, in the absence or presence of sulfate, gave more insight into the major players involved in the degradation of unsaturated and saturated LCFA. Syntrophomonas-related species were identified as predominant microorganisms in all the enrichment cultures. Microorganisms clustering within the family Syntrophobacteraceae were identified in the methanogenic and sulfate-reducing enrichments growing on palmitate. Distinct bacterial consortia were developed in oleate and palmitate enrichments, and observed differences might be related to the different degrees of saturation of these two LCFA. A new obligately syntrophic bacterium, Syntrophomonas zehnderi, was isolated from an oleatedegrading culture and its presence in oleate-degrading sludges detected by 16S rRNA gene cloning and sequencing.
\end{abstract}

Key words | anaerobic digestion, LCFA, oleate, palmitate, syntrophy

\section{INTRODUCTION}

Wastewaters, particularly those from food processing industries, contain considerable amounts of long-chain fatty acids (LCFA). These compounds, resulting from the hydrolysis of oils and fats, are potentially attractive for biogas production because of their high potential methane yield. Yet, removal of LCFA from the wastewaters prior to anaerobic treatment is rather standard, which implies the loss of heir energetic value. Reasons for this procedure are, basically, related with the recurrent reports on the alleged toxic/inhibitory effect of LCFA towards methanogenic activity (e.g. Hanaki et al. I98I; Koster \& Cramer 1987; Rinzema et al. 1994), as well as with problems of sludge flotation and washout during the doi: $10.2166 /$ wst.2008.090

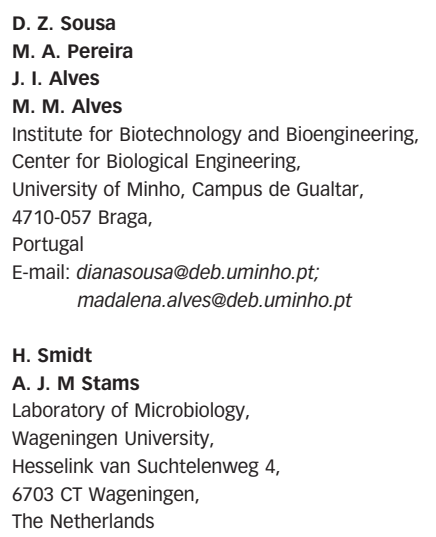

treatment of LCFA-rich wastewater in high-rate anaerobic reactors (e.g. Rinzema I988; Hwu et al. 1998a; Hwu et al. 1998b) (Figure 1A). However, studies conducted at our research group showed that the adverse effects of LCFA on anaerobic sludge functionality are not irreversible and that, under appropriate conditions, LCFA can be efficiently converted to methane (Pereira et al. 2003, 2004) (Figure 1B). Cycles of continuous feeding of lipid/LCFA-rich wastewaters followed by batch degradation of the accumulated substrate might be an appropriate way to treat this type of wastewater.

In methanogenic reactor systems, LCFA degradation proceeds via $\beta$-oxidation, yielding acetate and hydrogen, 


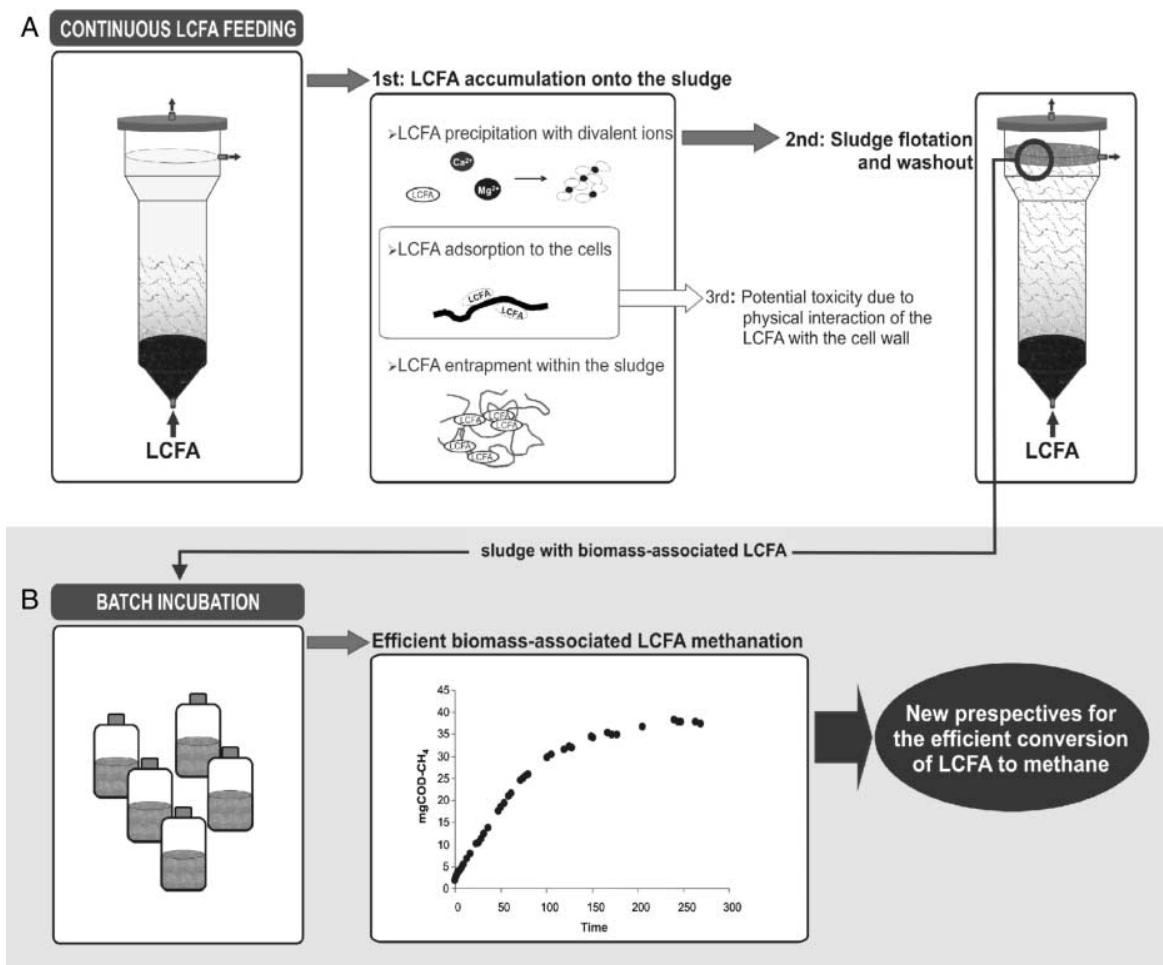

Figure 1 | Flow chart of the sequential LCFA degradation. (A) When a lipid/LCFA-rich wastewater is fed to a continuous anaerobic reactor, a substantial accumulation of LCFA onto the sludge is observed. LCFA accumulation is progressive and, at long last, conversion to methane stops and the sludge, then enclosed by a whitish foam, starts to float and to washout from the reactor. These operational problems, associated with the theories of LCFA toxicity towards anaerobic communities, required lipids/LCFA removal from wastewaters before biological treatment. (B) Batch incubation of the LCFA-"loaded" sludge, without addition of other carbon or energy sources, demonstrated that this sludge is actually still able to convert the biomass-associated LCFA to high amounts of methane. These results contradict the findings about the severe and irreversible toxicity of LCFA and suggest that the apparent inhibition during continuous LCFA feeding is reversible. New perspectives for the efficient conversion of LCFA to methane, potentially based on a two-phase process (LCFA continuous accumulation followed by batch degradation), are prospected from these new insights.

which are subsequently converted to methane and $\mathrm{CO}_{2}$ (Weng \& Jeris 1976). The overall conversion involves the concerted action of LCFA-oxidizing bacteria and methanogenic archaea that utilize hydrogen and acetate (Schink 1997). In environments where sulfate is present sulfate-reducing bacteria can oxidize LCFA to acetate and $\mathrm{CO}_{2}$ (or ultimately only to $\mathrm{CO}_{2}$ ), with production of sulfide (Rabus et al. 2000). Thus far, 10 acetogenic bacteria have been characterized that grow on fatty acids with more than 4 carbon atoms and up to 18 carbon atoms, in syntrophic association with methanogens. They all belong to the families Syntrophomonadaceae within the group of low $\mathrm{G}+\mathrm{C}$-containing Gram-positive bacteria (McInerney 1992; Zhao et al. 1993; Wu et al. 2006), or Syntrophaceae in the subclass of the $\delta$-Proteobacteria (Jackson et al. 1999). Described sulfate-reducing LCFA-oxidizers are relatively more diverse and are distributed among 13 different genera within the Desulfobacterales, Desulfuromonadales and Syntrophobacterales orders (Rabus et al. 2000).
Current knowledge on LCFA degradation is mainly derived from the study of pure cultures. A better understanding of the microbial diversity and function of LCFA-degrading communities in anaerobic reactors is still lacking. Insight about the phylogenetic affiliation of microorganisms involved in LCFA degradation can be investigated by molecular $16 \mathrm{~S}$ rRNA gene-targeting techniques, which in combination with cultivation techniques might give a more detailed picture of the microbial communities directly involved in LCFA degradation. Linking microbial information with biotechnological advances might be crucial for the development of new approaches enabling the efficient treatment of LCFA-rich wastewaters. Hence, this paper reviews some of the results obtained at our research group concerning microbiological aspects of the degradation of LCFA in anaerobic environments. Oleate and palmitate, the most abundant unsaturated and saturated LCFA present in wastewaters, respectively, were used as model substrates throughout the research. 


\section{LCFA-DEGRADING MICROBIAL COMMUNITIES IN METHANOGENIC REACTORS}

Microbial communities present in sludge samples submitted to a cycle of continuous LCFA-feeding followed by batch degradation of biomass-associated substrate were studied by using molecular techniques. A total of five sludge samples were compared: samples containing a high amount of accumulated LCFA, SO and SP, obtained after a continuous load in two EGSB reactors with oleate (unsaturated LCFA) and palmitate (saturated LCFA), respectively; the suspended sludge used as inoculum for both reactors, sample I; and samples $\mathrm{SO}_{\mathrm{b}}$ and $\mathrm{SP}_{\mathrm{b}}$, obtained after batch incubation of sludge $\mathrm{SO}$ and $\mathrm{SP}$, respectively, to allow the degradation of the accumulated LCFA. Predominant bacterial and archaeal phylotypes of the different samples were monitored using DGGE of PCRamplified 16S rRNA gene fragments (Figure 2). Composition of the predominant community visualized in the DGGE patters was determined by $16 \mathrm{~S}$ rRNA gene sequencing of 22 clones (bands 1-22 Figure 2, Table 1).

Comparison of the DGGE band-patterns from sludge samples I, SO and SP revealed a significant shift in the composition of the bacterial community during the continuous load with LCFA (accumulation step). At the end of the continuous feeding, the bacterial community present in the reactors fed with oleate and palmitate exhibited a low resemblance (54\% Pearson similarity between SO and SP). No significant changes in the archaeal communities were observed during LCFA accumulation, indicating that composition of the archaeal microbiota in the bioreactor remained rather stable (99\% Pearson similarity between SO and SP). After batch degradation of the accumulated substrates changes in the bacterial profiles were observed with Pearson similarity indices for sludge samples $\mathrm{SO} / \mathrm{SO}_{\mathrm{b}}$ and $\mathrm{SP} / \mathrm{SP}_{\mathrm{b}}$ of 61 and $75 \%$, respectively. Archaea profiles of both depleted sludges exhibited a lower similarity towards the respective LCFAloaded sludge as compared to the bacterial microbiota (archaeal profile Pearson similarity indices for $\mathrm{SO} / \mathrm{SO}_{\mathrm{b}}$ and $\mathrm{SP} / \mathrm{SP}_{\mathrm{b}}$ were 37 and $6 \%$, respectively).

In the overall LCFA-accumulation/degradation process a majority of the analyzed bacterial clones (87\%) clustered within the Firmicutes phylum. A prevalence of microorganisms belonging to the Clostridiaceae (Figure 2, bands 1, 2, 4, 5 and 14) and Syntrophomonadaceae (Figure 2, bands 6, 10, 11 and 15) suggests that these populations play an important role in LCFA degradation. Also relevant is the fraction of the retrieved $16 \mathrm{~S}$ rRNA gene sequences that are closely related to yet uncultured microorganisms (53\% of the total sequences), suggesting that more work on the cultivation of LCFAdegrading bacteria should be done. Hydrogenotrophic archaea, liable to make the overall LCFA conversion thermodynamically favorable, were present in all the
A

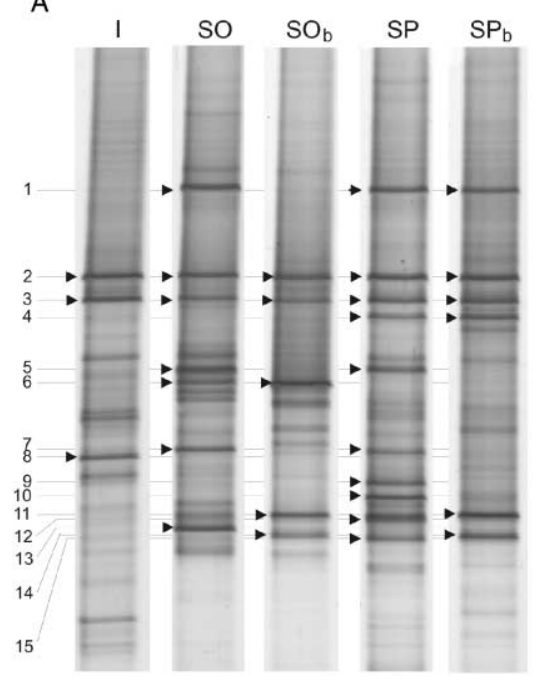

B

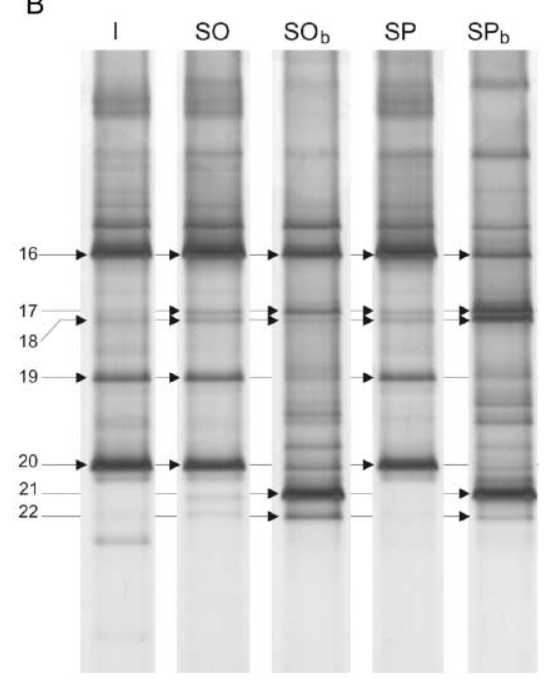

Figure 2 | DGGE patterns of (A) bacterial and (B) archaeal amplicons obtained from the sludge samples: I - inoculum, SO-sludge after continuous load in EGSB reactor with oleate, $\mathrm{SP}$ - sludge after continuous load in EGSB reactor with palmitate, $\mathrm{SO}_{\mathrm{b}}$-sludge $\mathrm{SO}$ after degradation of accumulated $\mathrm{LCFA}$ in batch, $\mathrm{SP}_{\mathrm{b}}-\mathrm{Sludge} \mathrm{SP}$ after degradation of accumulated LCFA in batch. 
Table 1 Affiliation of the retrieved bacterial (clones 1 to 15) and archaeal (clones 16 to 22) clones

\begin{tabular}{|c|c|c|c|c|}
\hline Band ID & Closest relative (>1200 bp) & $\%$ Identity & Phylum & Accession nos. \\
\hline 1 & Clostridium sp 45 & 99 & Firmicutes & DQ339700 \\
\hline 2 & Clostridium butyricum & 99 & Firmicutes & DQ339701 \\
\hline 3 & Uncultured bacterium clone $\mathrm{C} 118$ & 96 & Firmicutes & DQ339702 \\
\hline 4 & Uncultured bacterium clone p-2117-s959-2 & 99 & Firmicutes & DQ339703 \\
\hline 5 & Clostridium propionicum & 99 & Firmicutes & DQ339704 \\
\hline 6 & Uncultured bacterium clone R6b2 & 94 & Firmicutes & DQ339705 \\
\hline 7 & Uncultured bacterium $\mathrm{H} 30$ & 96 & Proteobacteria & DQ339706 \\
\hline 8 & Eubacterium callanderi & 89 & Firmicutes & DQ339707 \\
\hline 9 & Uncultured bacterium Eub No 20 & 97 & Firmicutes & DQ339708 \\
\hline 10 & Unidentified eubacterium clone vadinCA02 & 99 & Firmicutes & DQ339709 \\
\hline 11 & Syntrophomonas wolfei & 93 & Firmicutes & DQ339710 \\
\hline 12 & Uncultured bacterium clone TSAT05 & 99 & Firmicutes & DQ339711 \\
\hline 13 & Uncultured bacterium clone PL-7B6 & 98 & Bacteroidetes & DQ339712 \\
\hline 14 & C.butyricum (NCIMB8082) & 94 & Firmicutes & DQ339713 \\
\hline 15 & Syntrophomonas wolfei & 92 & Firmicutes & DQ339714 \\
\hline 16 & Methanobacterium formicicum strainFCam & 98 & Euryarchaeota & DQ339715 \\
\hline 17 & Methanobacterium aarhusense & 97 & Euryarchaeota & DQ339716 \\
\hline 18 & Methanobacterium formicicum strainFCam & 98 & Euryarchaeota & DQ339717 \\
\hline 19 & Methanosaeta concilii & 99 & Euryarchaeota & DQ339718 \\
\hline 20 & Methanosaeta concilii & 99 & Euryarchaeota & DQ339719 \\
\hline 21 & Methanosarcina mazei strain Goe1 & 99 & Euryarchaeota & DQ339720 \\
\hline 22 & Methanosarcina mazei strain Goe1 & 99 & Euryarchaeota & DQ339721 \\
\hline
\end{tabular}

analyzed samples. Members of the Methanobacterium genus are predominant in both accumulation and degradation steps (Figure 2, bands 16, 17 and 18). Methanosaeta spp were detected as predominant acetate-utilizers during continuous oleate and palmitate feeding (Figure 2, bands 19 and 20) but, were virtually replaced by Methanosarcina spp after batch degradation of the accumulated substrate (Figure 2, bands 21 and 22). This might be related with differences in the acetate concentration during the continuous feeding and batch degradation. In fact, acetate concentrations in the continuous reactors were rather low (below $300 \mathrm{mg} \mathrm{L}^{-1}$ ), favoring the dominance of Methanosaeta spp that have a high affinity for acetate (Jetten et al. 1992). On the other hand, during batch degradation, the release of large amounts of acetate to the medium due to degradation of the biomass-associated LCFA might create favorable conditions for growth of Methanosarcina spp, which have a lower affinity for acetate but a higher growth rate than Methanosaeta spp (Jetten et al. I992).

\section{MICROBIAL COMMUNITIES PRESENT IN OLEATE- AND PALMITATE-ENRICHMENT CULTURES}

LCFA-degrading communities were developed by selective enrichments growing on oleate (unsaturated LCFA) and palmitate (saturated LCFA), in the presence and absence of sulfate. The same inoculum sludge was used to start up four different enrichment series: $\mathrm{OM}$ and PM cultures, growing on oleate or palmitate, respectively, without the presence of a inorganic electron acceptor other than protons and $\mathrm{CO}_{2}$ (favoring methanogenesis) and, OS and PS cultures, growing on oleate or palmitate, respectively, in the presence of sulfate (favoring sulfate reduction). Later on, stable methanogenic $\mathrm{OM}$ and $\mathrm{PM}$ were incubated in medium containing oleate or palmitate, respectively, plus sulfate, and subjected to subsequent transfers with sulfate: OM-OS and PM-PS cultures. Changes in the microbial composition during enrichment were analyzed by DGGE profiling 


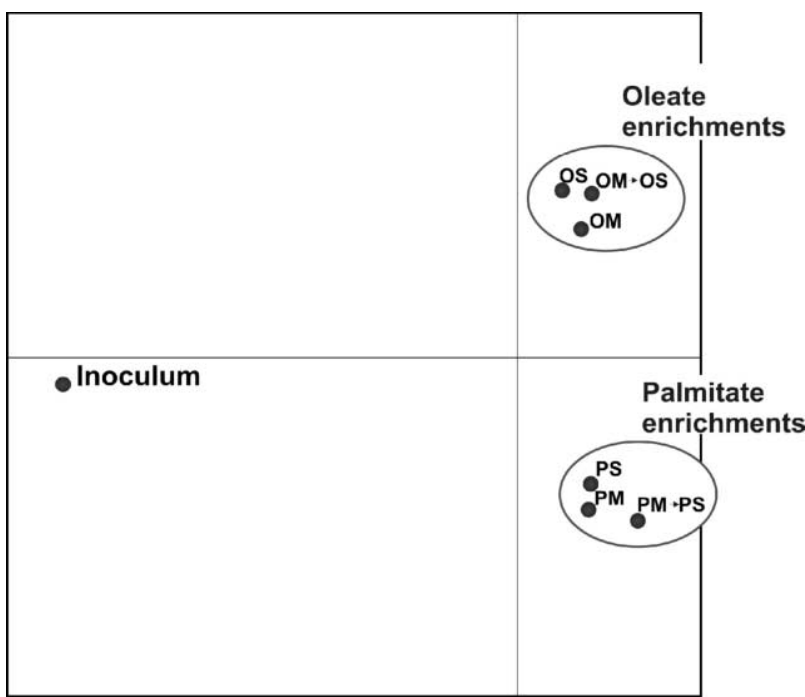

Figure 3 | PCA of DGGE profiles obtained after specific 16S rRNA gene amplification of genomic DNA from stable oleate- and palmitate-enrichment cultures: OM-methanogenic oleate-enrichment (12 transfers); PM-methanogenic palmitate-enrichment (12 transfers); OS-sulfate-reducing oleate-enrichment (7 transfers); PS-sulfate-reducing palmitate-enrichment (7 transfers); $\mathrm{OM} \rightarrow$ OS-methanogenic oleate-enrichment after 4 successive transfers in medium containing sulfate; PM $\rightarrow$ PS-methanogenic palmitate-enrichment culture after 4 successive transfers in medium containing sulfate. PC1 and PC2 represent $40.3 \%$ and $30.5 \%$ of the variation, respectively.

of PCR-amplified 16S rRNA gene fragments (data not shown). DGGE profiles of stable enrichment cultures were treated by principal component analysis (PCA) to evaluate distances between the different communities (Figure 3). As result of the PCA analysis two marked clusters, relatively distant from the inoculum sludge, were obtained: one cluster containing the different oleate-enrichment cultures and the other with the palmitate-enrichment cultures. These results suggest that the substrate used during enrichment had a major influence in the bacterial community structure

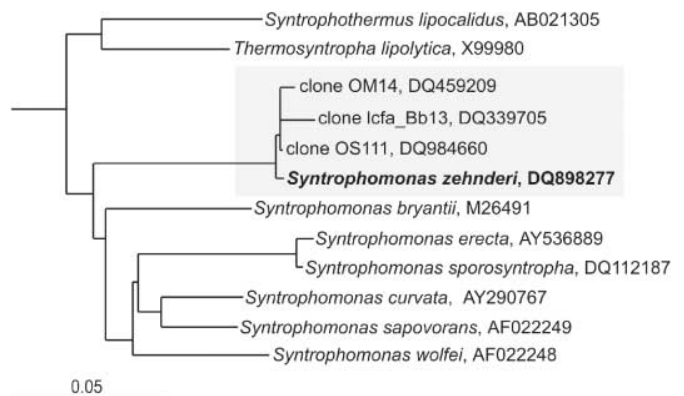

Figure 4 Phylogenetic tree showing the position of the 16S rRNA gene clones retrieved from the different sludge samples in contact with oleate (DQ339705, DQ459209 and DQ98466) within representatives of fatty acid degrading bacteria. and a stronger shifting effect than the presence or absence of sulfate in the environment. One reason that might partially explain the differential clustering of the bacterial populations present in oleate- and palmitate-enrichment cultures is the fact that the two LCFA used have different degree of chain saturation. Oleate is an unsaturated LCFA with a double bond at position C9, while palmitate has a completely saturated chain. In fact, only a minority of the characterized LCFA-degrading bacteria is able to degrade unsaturated LCFA, indicating that this is a specific feature of some microorganisms (Sousa 2007).

Prominent DGGE-bands of the enrichment cultures were identified by $16 \mathrm{~S}$ rRNA gene sequencing. A significant part of the retrieved $16 \mathrm{~S}$ rRNA gene sequences was most similar to those of uncultured bacteria. 16S rRNA gene sequences clustering within the Syntrophomonadaceae family were identified as corresponding to predominant DGGE-bands in the oleate- and palmitate-enrichment cultures (OM, OS, $\mathrm{PM}$ and PS). In both stable palmitate-enrichment cultures (PM and PS) members of the Syntrophobacteraceae family were present. In the enrichment cultures grown in the presence of sulfate (OS and PS) sulfate-reducing bacteria closely affiliated with Desulfovibrio, Desulfomicrobium and Desulforhabdus genera were also detected. These bacteria are probably involved in the use of the hydrogen and acetate resulting from LCFA oxidation, in a similar role taken by methanogenic archaea when no sulfate is present in the medium.

\section{Syntrophomonas zehnderi, a novel syntrophic LCFA- degrading bacterium linked to oleate degradation}

A novel LCFA-degrading bacterium, Syntrophomonas zehnderi, was isolated as a co-culture with Methanobacterium formicicum from an anaerobic bioreactor treating an oleate-based effluent (Sousa et al. 2007). This mesophilic, syntrophic, fatty acid oxidizing bacterium degrades straight-chain fatty acids with 4 to 18 carbon atoms but, also, unsaturated LCFA, such as oleate (in co-culture with $M$. formicicum). 16S rRNA gene sequences affiliated with Syntrophomonas zehnderi were retrieved from sludges degrading oleate under different conditions, i.e. continuous load, fed-batch operation and enrichment series (Figure 4). The presence of Syntrophomonas zehnderi related bacteria in sludges after contact with oleate suggests its important 
role in anaerobic oleate degradation in bioreactor sludge. Moreover, the fact that these microorganisms are present in the oleate-degrading sludges but not in palmitate-degrading sludges suggests their direct link to oleate degradation.

\section{$\overline{\text { CONCLUSIONS }}$}

Application of cultivation and molecular techniques to the study of microbial composition of LCFA-degrading sludges provided important insight into the communities involved in the degradation of these compounds. Members of the Clostridiaceae and Syntrophomonadaceae appeared pivotal to LCFA-degradation. Oleate- and palmitate-degrading cultures showed a different microbial composition, indicating that the types of bacteria in the community might depend on the saturation degree of the fed LCFA. A novel LCFAdegrading bacterium, Syntrophomonas zehnderi, was isolated from an oleate-enrichment culture and could be linked to oleate degradation under several conditions. A proposal for genome sequencing of this bacterium is accepted by the DOEJGI and future genomic comparison of this strain with S. wolfei, which only degrades saturated LCFA, will provide more insight into the differences in the degradation of unsaturated and saturated LCFA.

\section{ACKNOWLEDGEMENTS}

The authors acknowledge the Fundação para a Ciência e Tecnologia (FCT) and Fundo Social Europeu (FSE) for the financial support given to D.Z. Sousa (SFRH/BD/8726/2002) and M. A. Pereira (SFRH/BPD/14591/2003).

\section{REFERENCES}

Hanaki, K., Nagase, M. \& Matsuo, T. I98I Mechanism of inhibition caused by long-chain fatty acids in anaerobic digestion process. Biotechnol. Bioeng. 23(7), 1591-1610.

Hwu, C.-S., van Lier, J. B. \& Lettinga, G. I998a Physicochemical and biological performance of expanded granular sludge bed reactors treating long-chain fatty acids. Process Biochem. 33(1), 75-81.

Hwu, C.-S., Tseng, S. K., Yuan, C. Y., Kulik, Z. \& Lettinga, G. I998b Biosorption of long-chain fatty acids in UASB treatment process. Water Res. 32(5), 1571-1579.

Jackson, B. E., Bhupathiraju, V. K., Tanner, R. S., Woese, C. R. \& McInerney, M. J. 1999 Syntrophus aciditrophicus sp. nov., a new anaerobic bacterium that degrades fatty acids and benzoate in syntrophic association with hydrogen-using microorganisms. Archives of Microbiol. 171(2), 107-114.

Jetten, M. S. M,, Stams, A. J. M, \& Zehnder, A. J. B. 1992 Methanogenesis from acetate: a comparison of the acetate metabolism in Methanothrix soehngenii and Methanosarcina spp. FEMS Microbiol. Rev. 88(3-4), 181-197.

Koster, I. W. \& Cramer, A. I987 Inhibition of methanogenesis from acetate in granular sludge by long-chain fatty acids. Appl. Environ. Microbiol. 53(2), 403-409.

McInerney, M. J. 1992 The genus Syntrophomonas, and other syntrophic bacteria. In: Balows, A., Trüper, H. G., Dworkin, M., Harder, W. \& Schleifer, K. H. (eds), The Prokaryotes. Springer, New York, pp. 2048-2057.

Pereira, M. A., Cavaleiro, A. J., Mota, M. \& Alves, M. M. 2003 Accumulation of long chain fatty acids onto anaerobic sludge under steady state and shock loading conditions: effect on acetogenic and methanogenic activity. Water Sci. Technol. 48(6), 33-40.

Pereira, M. A., Sousa, D. Z., Mota, M. \& Alves, M. M. 2004 Mineralization of LCFA associated with anaerobic sludge: kinetics, enhancement of methanogenic activity, and effect of VFA. Biotechnol. Bioeng. 88(4), 502-511.

Rabus, R., Hansen, T. \& Widdel, F. 2000 Dissimilatory sulfate- and sulfur-reducing prokaryotes. In: Dworkin, M., Falkow, S., Rosenberg, E., Schleifer, K. -H. \& Stackebrandt, E. (eds), The Prokaryotes: An Evolving Electronic Resource for the Microbiological Community. Springer-Verlag, New York.

Rinzema, A. 1988 Anaerobic treatment of wastewater with high concentrations of lipids or sulfate. Wageningen Agricultural University, The Netherlands.

Rinzema, A., Boone, M., van Knippenberg, K. \& Lettinga, G. 1994 Bactericidal effect of long chain fatty acids in anaerobic digestion. Water Environ. Res. 66, 40-49.

Schink, B. 1997 Energetics of syntrophic cooperation in methanogenic degradation. Microbiol. Mol. Biol. Rev. 61(2), 262-280.

Sousa, D. Z. 2007 Ecology and physiology of microbial communities that degrade long-chain fatty acids. University of Minho, Portugal.

Sousa, D. Z., Smidt, H., Alves, M. M. \& Stams, A. J. M. 2007 Syntrophomonas zehnderi sp. nov., an anaerobe that degrades long chain fatty acids in co-culture with Methanobacterium formicicum. Int. J. Syst. Evol. Microbiol. 57, 609-615.

Weng, C. \& Jeris, J. S. 1976 Biochemical mechanisms in methane fermentation of glutamic and oleic acids. Water Res. 10(1), $9-18$.

Wu, C., Liu, X. \& Dong, X. 2006 Syntrophomonas erecta subsp. sporosyntropha subsp. nov., a spore-forming bacterium that degrades short chain fatty acids in co-culture with methanogens. Syst. Appl. Microbiol. 29(6), 457-462.

Zhao, H., Yang, D., Woese, C. R. \& Bryant, M. P. 1993 Assignment of fatty acid-b-oxidizing syntrophic bacteria to Syntrophomonadaceae fam. nov. on the basis of $16 \mathrm{~S}$ rRNA sequence analysis. Int. J. Syst. Bacteriol. 43, 278-286. 